\section{Vitamin D}

\section{Vitamin $D$ nutrition is at a crossroads}

Madam

The Institute of Medicine's (IOM) latest recommendations $^{(1)}$ defining the formal RDA of vitamin $\mathrm{D}$ required for good health ( $15 \mu \mathrm{g}$ vitamin D for persons aged $1-70$ years and $20 \mu \mathrm{g}$ vitamin $\mathrm{D}$ for persons aged $>70$ years) are largely inconsequential, because the change from the 1997 IOM recommendations $(5-15 \mu \mathrm{g} / \mathrm{d}$, depending on age $)^{(2)}$ is so small. Also the IOM committee ignored the consensus of hundreds of vitamin $\mathrm{D}$ research scientists and nutritionists from at least twenty-five countries who attended the 13th Vitamin D Workshop in 2006 in Victoria, British Columbia, Canada ${ }^{(3)}$ and the14th Workshop in 2009 in Brugge, Belgium ${ }^{(4)}$. For a definition of one unit see footnote $\# 1$.

There are two major components of the consensus of these scientists. First, research over the past decade has resulted in the addition of four physiological systems to vitamin D's responsibilities, acting through the steroid hormone $1 \alpha, 25-(\mathrm{OH})_{2}$ vitamin $\mathrm{D}$, for good health maintenance and disease prevention, including: (i) the immune system (both innate and adaptive); (ii) the cardiovascular system; (iii) muscle; and (iv) the pancreas and metabolic homeostasis $^{(5)}$. Second, it is generally agreed that in North America and Western Europe half of the elderly population is vitamin D-deficient; in the rest of the world, about twothirds of the total population does not receive adequate amounts of the vitamin to even maintain healthy bone ${ }^{(3)}$.

Both governmental agencies worldwide and individuals are now at a nutritional crossroads with respect to choosing their appropriate vitamin D intake. There are two choices. Both governments and individuals can accept the very conservative advice of the IOM focused on bone health and forgo the benefits to good health that could accrue with a higher daily intake of vitamin D. Or we can, acting as individuals, become informed about and make our own decisions regarding our personal daily intake of vitamin D. When I am asked for my advice, I suggest (in congruence with many vitamin $\mathrm{D}$ scientists), a vitamin $\mathrm{D}$ intake of $50-100 \mu \mathrm{g} / \mathrm{d}$ for adults. This is stated to be a 'tolerable dose' e.g. safe by the current IOM report. Research strongly suggests that a lifetime vitamin D intake at this safe level would prevent borderline vitamin D deficiency, reduce many diseases, increase the longevity and quality of life, and diminish medical care costs worldwide ${ }^{(5)}$.

How can people acquire this dose? Under the right circumstances exposure to sunlight can generate significant amounts of the vitamin, but this method has two drawbacks. First, sunlight exposure can result in skin cancer as well as non-lethal skin damage ${ }^{(6)}$. Second, approximately one-third of the world's citizens $\left(2 \cdot 3\right.$ billion) live between $40^{\circ} \mathrm{N}$ and

\footnotetext{
${ }^{1}$ One International unit (IU) $=0.025$ micrograms or 25 nanograms. Thus
} one microgram of Vitamin D $=40 \mathrm{IU}$.
Table 1 Serum 25-hydroxyvitamin D $(25(\mathrm{OH}) \mathrm{D})$ levels define a person's vitamin D status (modified from Norman and Bouillon ${ }^{(5)}$ )

\begin{tabular}{llll}
\multicolumn{2}{c}{ Serum 25(OH)D } & \\
\cline { 1 - 1 } $\mathrm{ng} / \mathrm{ml}$ & $\mathrm{nmol} / \mathrm{l}$ & & Nutritional descriptor \\
\hline$<5$ & $<12$ & & Severe vitamin D deficiency \\
$5-10$ & $12-25$ & & Vitamin D deficiency \\
$10-20$ & $25-50$ & & Vitamin D insufficiency \\
$20-30$ & $50-75$ & & Marginal vitamin D status \\
$30-60$ & $75-150$ & & Vitamin D sufficiency \\
$>150$ & $>375$ & Risk for toxicity \\
\hline
\end{tabular}

$90^{\circ} \mathrm{N}$ where, for a significant portion of the year, the amount and intensity of sunshine is inadequate.

Is it better to provide proper vitamin D supplements or to fortify food with vitamin D? Unfortified foods with useful amounts of vitamin $\mathrm{D}$ are rare, the best sources being animal products such as fatty fish and liver extracts (codliver oil). In the USA, the Food and Drug Administration has approved the fortification of milk and milk products, breakfast cereal, orange juice, pastas, infant formulas and margarines. In third-world countries reliable sources of vitamin D-enriched food are often entirely lacking. Thus, inexpensive forms of vitamin D supplementation need to be made available in the correct dosage range.

The failsafe remedy for concerned citizens, therefore, is personal vitamin D supplementation. Inexpensive capsules are available for adults to achieve an intake of $50-100 \mu \mathrm{g} / \mathrm{d}$. Both the 1998 and 2010 IOM committees and many other concerned scientists believe that an individual's vitamin D nutritional status should be determined by carrying out serum assays for 25-hydroxyvitamin D [25(OH)D]. Table 1 provides a sequential series of six guidelines concerning serum 25(OH)D levels as a measure of relative vitamin $\mathrm{D}$ nutritional status: (i) severe vitamin D deficiency; (ii) vitamin D deficiency; (iii) vitamin D insufficiency; (iv) marginal vitamin D status; (v) vitamin D sufficiency; and (vi) risk for toxicity. The serum $25(\mathrm{OH}) \mathrm{D}$ levels that define the first three categories are also endorsed by the 2010 IOM committee. The author and many other scientists in the field believe that the range of $20-30 \mathrm{ng} / \mathrm{ml}$ is a state of marginal vitamin D status and that, to ensure an adequate response by the calcium homeostatic system as well as the four new biological systems, it is essential to have achieved a state of 'vitamin D sufficiency'; this is a serum 25(OH)D concentration in the range of $30-60 \mathrm{ng} / \mathrm{ml}(75-150 \mathrm{nmol} / \mathrm{l})$.

Thus an annual physical examination should include a determination of the blood level of $25(\mathrm{OH}) \mathrm{D}$, which should fall in the range of $30-60 \mathrm{ng} / \mathrm{ml}$; see Table 1 . Maintained consistently, such a vitamin D blood level will ensure good bone health and, at the same time, help realize the vitamin's wide range of new-found benefits.

Anthony W. Norman Department of Biochemistry and Division of Biomedical Sciences 
Room 5456 Boyce Hall University of California

Riverside, CA 92521 USA

Email: Anthony.norman@ucr.edu doi:10.1017/S1368980011000280

\section{References}

1. Institute of Medicine (2011) Dietary Reference Intakes for Calcium and Vitamin D. Washington, DC: National Academies Press.

2. Institute of Medicine (1997) Dietary Reference Intakes for Calcium, Magnesium, Phosphorus, Vitamin D, and Fluoride. Washington, DC: National Academies Press, pp. 250-287.

3. Norman AW, Bouillon R, Whiting SJ et al. (2007) 13th Workshop consensus for vitamin D nutritional guidelines. J Steroid Biochem Mol Biol 103, 204-205.

4. Henry HL, Bouillon R, Norman AW et al. (2010) 14th Vitamin D Workshop consensus on vitamin D nutritional guidelines. J Steroid Biochem Mol Biol 121, 4-6.

5. Norman AW \& Bouillon R (2010) Vitamin D nutritional policy needs a vision for the future. Exp Biol Med $\mathbf{2 3 5}$, 1034-1045.

6. Gilchrest BA (2007) Sun protection and vitamin D: three dimensions of obfuscation. J Steroid Biochem Mol Biol 103, 655-663.

\section{Vitamin D}

The Institute of Medicine did not find the vitamin D-cancer link because it ignored UV-B dose studies

\section{Madam}

When The Institute of Medicine (IOM) of the National Academies released its new Dietary Reference Intakes for Calcium and Vitamin D report on 30 November $2010^{(1,2)}$, the vitamin D research community was shocked and dismayed at the findings. The committee found a benefit only for bones, leading to the finding that a 25-hydroxyvitamin D (25(OH)D) level of $20 \mathrm{ng} / \mathrm{ml}$ was adequate and a recommended intake of $15 \mu \mathrm{g} / \mathrm{d}$ for most people. These are well below the recommendations of vitamin $\mathrm{D}$ experts: intakes of up to $50 \mu \mathrm{g} / \mathrm{d}$ and achieving serum $25(\mathrm{OH}) \mathrm{D}$ levels of $40-60 \mathrm{ng} / \mathrm{ml}^{(3)}$. Casual solar UV-B irradiance in summer in England raises serum 25(OH)D levels by nearly $40 \mathrm{nmol} / 1$, equivalent to the production of about $37.5 \mu \mathrm{g} / \mathrm{d}$ for those aged 45 years $^{(4)}$, far more than suggested by the $\operatorname{IOM}^{(1)}$.

The UV-B-vitamin D-cancer hypothesis was based on an ecological study of the geographical variation of colon cancer mortality rates and sunlight doses in the USA $^{(5)}$ and has been extended by subsequent ecological studies in Australia, Asia, Europe and the USA to about twenty types of cancer ${ }^{(6-9)}$. While the IOM considered some ecological studies as background information, it noted they have the primary weakness that 'Outcome measures are not predictable at the individual level' and, thus, are of low quality for dietary reference intakes ${ }^{(1)}$. This summary dismissal is not warranted: in part because no mechanism other than production of vitamin $D$ has been proposed to explain the ecological study findings, in part since the findings of ecological studies of cancer have been supported by other studies ${ }^{(10)}$, and in part since ecological studies integrate the effect of UV-B and vitamin $\mathrm{D}$ over much of the lifetime and include many cases.

A second type of study based on solar UV-B is that of cancer risk with respect to diagnosis or death from non-melanoma skin cancer (NMSC). The primary risk factor for NMSC is UV irradiance, with UV-B the most important risk factor for NMSC death ${ }^{(11)}$. An ecological study for Spain found fifteen types of cancer inversely correlated with NMSC mortality rate after adjusting for smoking ${ }^{(12)}$. A record linkage study found significant inverse correlations between diagnosis of NMSC and incidence of gastric, liver, pancreatic and prostate cancer and non-significant inverse correlations for five other types of cancer $^{(13)}$. A reduced risk of prostate cancer incidence was noted with more early-life UV-B irradiance ${ }^{(14)}$.

A third type of study is based on solar UV-B exposure related to occupation. A death certificate-based casecontrol study of cancer mortality rates in the USA found significant inverse correlations for breast and colon cancer with respect to occupations with high occupational exposure to sunlight ${ }^{(15)}$. A study of cancer risk in Rhineland-Palatinate, Germany found significantly reduced risk of nearly a dozen types of internal cancer compared with incidence of NMSC plus melanoma in regions with more land devoted to winegrowing ${ }^{(16,17)}$.

A fourth type of study is the case-control study using self-reported personal sun exposure. A pooled study of this nature found a protective effect of recreational sun exposure at 18-40 years of age and in the 10 years before diagnosis for non-Hodgkin's lymphoma ${ }^{(18)}$.

Together with other studies such as case-control studies of vitamin D and breast cancer ${ }^{(19)}$ and improved survival rate after diagnosis of non-Hodgkin's lymphoma and other types of cancer with higher serum $25(\mathrm{OH}) \mathrm{D}$ at time of diagnosis $^{(20)}$, there is strong support for a causal relationship between vitamin D and reduced risk of cancer ${ }^{(10)}$ which could have permitted the IOM to find a beneficial effect of vitamin $\mathrm{D}$ in reducing the risk of cancer.

\section{Disclosure}

I receive or have received funding from the UV Foundation (McLean, VA, USA), the Sunlight Research Forum (Veldhoven, The Netherlands), Bio-Tech-Pharmacal (Fayetteville, AR, USA), the Vitamin D Council (San Luis Obispo, CA, USA) and the Danish Sunbed Federation (Middelfart, Denmark).

William B. Grant Sunlight, Nutrition, and Health Research Center (SUNARC)

PO Box 641603

San Francisco, CA 94164-1603, USA Email: wbgrant@infionline.net doi:10.1017/S1368980011000267 